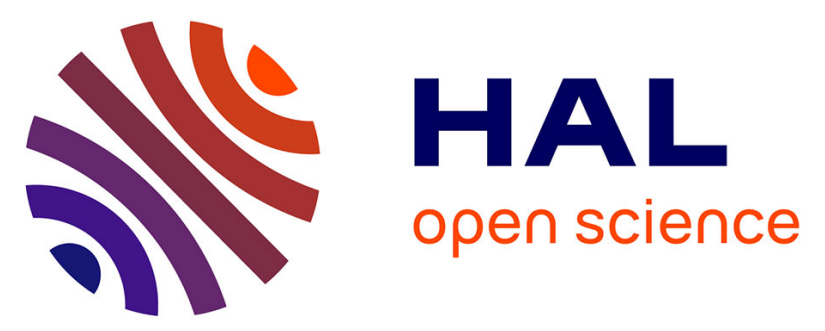

\title{
DETECTION AND ESTIMATION OF DELAYS IN BIVARIATE SELF-SIMILARITY: BOOTSTRAPPED COMPLEX WAVELET COHERENCE
}

G. Didier, H. Wendt, Patrice Abry

\section{- To cite this version:}

G. Didier, H. Wendt, Patrice Abry. DETECTION AND ESTIMATION OF DELAYS IN BIVARIATE SELF-SIMILARITY: BOOTSTRAPPED COMPLEX WAVELET COHERENCE. IEEE International Conference on Acoustics, Speech and Signal Processing (ICASSP), May 2019, Brighton, United Kingdom. hal-02346773

\section{HAL Id: hal-02346773 \\ https://hal.science/hal-02346773}

Submitted on 7 Nov 2019

HAL is a multi-disciplinary open access archive for the deposit and dissemination of scientific research documents, whether they are published or not. The documents may come from teaching and research institutions in France or abroad, or from public or private research centers.
L'archive ouverte pluridisciplinaire HAL, est destinée au dépôt et à la diffusion de documents scientifiques de niveau recherche, publiés ou non, émanant des établissements d'enseignement et de recherche français ou étrangers, des laboratoires publics ou privés. 


\title{
DETECTION AND ESTIMATION OF DELAYS IN BIVARIATE SELF-SIMILARITY: BOOTSTRAPPED COMPLEX WAVELET COHERENCE
}

\author{
G. Didier ${ }^{1}, H$. Wendt ${ }^{2}$, P. Abry ${ }^{3}$ \\ ${ }^{1}$ Math. Dept., Tulane University, New Orleans, USA. \\ 2 IRIT, CNRS (UMR 5505), Université de Toulouse, France. \\ ${ }^{3}$ Univ Lyon, Ens de Lyon, Univ Claude Bernard, CNRS, Laboratoire de Physique, F-69342 Lyon, France.
}

\begin{abstract}
The self-similarity paradigm enables the analysis of scale-free temporal dynamics and has been widely used in a large set of real-world applications. However, in a multivariate setting, delays amongst components significantly impair the estimation of scale-free parameters. The first framework for the modeling, detection and estimation of delay parameters and for the joint estimation of scale-free parameters is proposed here. It is assumed that a single realization of a multivariate, self-similar time series is available. Use is made of $\mathbb{C}$-valued wavelets and, based on the imaginary part of the wavelet coherence, an original bootstrap-based delay estimation procedure based is constructed. Moreover, a consistent wavelet eigenanalysisbased semiparametric estimation for scale-free parameters that accounts for delay is defined. Monte Carlo experiments conducted over various instances of the model show that the proposed methodology enables the detection of delays with high probability and provides very satisfactory estimates of the delay and scale-free parameters.
\end{abstract}

Index Terms - multivariate self-similarity, delay, complex wavelet coherence, bootstrap.

\section{INTRODUCTION}

Context: Multivariate self-similarity. Self-similarity [1] provides a framework for describing and modeling scale-free temporal dynamics, and it has been used with great success in a diverse range of real world applications (see, e.g., [2-4] and references therein). Fractional Brownian motion ( $\mathrm{fBm})$, the quintessential scale-free model, is the only Gaussian, stationary-increment stochastic process [5]. It underpins a robust and versatile data modeling paradigm where the dynamics are mainly driven by a single self-similarity parameter $0<H<1$. Knowledge of $H$ allows carrying out various classical signal processing tasks such as characterization, diagnosis, classification, detection. . Accordingly, the central practical task is to estimate $H$. Though several estimation procedures are available, it has now been well documented that wavelet analysis provides a theoretically well-grounded, robust and accurate methodology for the estimation of the parameter $H[2,6]$.

Nevertheless, the success of self-similarity modeling has mostly remained constrained to the univariate context. In the modern era of data deluge, data are often recorded as several joint time series. This generally renders univariate-like methods inappropriate and calls for the construction of modeling protocols that are inherently multivariate. Operator fractional Brownian motion (ofBm)

Work supported by French ANR BLANC MULTIFRACS. G.D. was partially supported by ARO grant W911NF-14-1-0475 and by a CNRS invited position at ENS-Lyon. was recently proposed as a model for multivariate self-similarity [7-10]. In essence, ofBm consists of a mixture of correlated $\mathrm{fBm}$ processes, each with possibly distinct self-similarity exponents $H_{m}$, $m=1, \ldots, M$. In $[4,11]$, a wavelet eigenvalue regression methodology is constructed and studied for the joint estimation of $\underline{H}=$ $\left(H_{1}, \ldots, H_{M}\right)$. OfBm has been successfully applied in many fields such as in Internet traffic modeling [4] and dendrochronology [12].

Related work: Time irreversibility and delay. The modeling and analysis of multivariate self-similarity has remained constrained by the assumption of time reversibility. A stochastic process $Y$ is called time reversible when its finite-dimensional distributions satisfy the equality $\{Y(-t)\}_{t \in \mathbb{R}} \stackrel{\text { fdd }}{=}\{Y(t)\}_{t \in \mathbb{R}}$. When $Y$ is a multivariate Gaussian, stationary-increment process, time reversibility implies that the matrix-valued spectral density $\Gamma_{Y}(f)$ of $Y$ is entrywise real for almost every frequency $f$ [9]. However, in many real world situations, data spectra display features - e.g., non-trivial phases - only appearing in a multivariate context and breaking time reversibility. Notably, delays amongst components constitute a simple, common and plausible cause for time irreversibility in real-world data, and must thus be taken into account when analyzing multivariate scale-free dynamics. In neuroscience, for instance, it is well documented that the assessment of brain functional connectivity is only meaningful for components with non-zero delays. These are due to physiological constraints and to the so-called volume conduction effects, caused by the propagation of information from a source to multiple sensors $[13,14]$. Hence, neuroscientific data modeling requires the reliable detection of non-zero delays and the accurate estimation of the joint parameters for delayed components. Accordingly, the estimation of parameters associated with time irreversibility has received considerable attention recently [15-21].

Goal and contributions. In the present work, we put forward a framework for the detection of delay amongst components in a multivariate scale-free dynamics context as well as for the joint estimation of delay and self-similarity exponents. We focus on the bivariate setting for simplicity. In Sec. 2, we recap the definition of classical ofBm and wavelet eigenvalue-based estimation of $\underline{H}$. In Sec. 3, we define the proposed time irreversible and asymptotically self-similar bivariate model. We also detail the methods for delay detection and for the joint estimation of delays and $\underline{H}$. Starting from complex-valued wavelet coefficients, this involves the combined use of the imaginary part of the wavelet coherence function, of a Fisher $z$-transform and of a wavelet domain multivariate bootstrap procedure. In Sec. 4, Monte Carlo simulations assess the practical relevance and statistical performance of the proposed procedures, both with respect to delay and to correlation amongst components. Random process synthesis and estimation are carried out by means of MATLAB routines, made available at the time of publication. 


\section{TIME REVERSIBLE BIVARIATE SELF-SIMILARITY}

OfBm. Hereinafter, we make use of a restricted definition of ofBm that implies time reversibility but is satisfactory for practical purposes. Readers are referred to [9] for a general definition. Let $X \equiv\left\{X_{H_{1}}(t), X_{H_{2}}(t)\right\}_{t \in \mathbb{R}}$ be a pair of possibly correlated fBms with individual self-similarity exponents $\underline{H}=\left(H_{1}, H_{2}\right), 0<H_{1} \leq$ $H_{2}<1$. The entries of the pointwise covariance matrix $\Sigma_{X}=$ $\mathbb{E} X(1) X(1)^{*}$ are given by $\left(\Sigma_{X}\right)_{m, m^{\prime}}=\sigma_{m} \sigma_{m^{\prime}} \rho_{m, m^{\prime}}$, with $\sigma_{1}^{2}$, $\sigma_{2}^{2}$ and $\rho \equiv \rho_{1,2}$ denoting the variances of each component and their respective correlation coefficients. Let $W$ be a $2 \times 2$, realvalued, invertible matrix. Bivariate ofBm $Y$ is defined as (in short, $Y=W X)$ :

$$
\left\{Y_{1}^{\underline{H}, \Sigma_{X}, W}(t), Y_{2}^{\underline{H}, \Sigma_{X}, W}(t)\right\}_{t \in \mathbb{R}}=W\left\{X_{H_{1}}(t), X_{H_{2}}(t)\right\}_{t \in \mathbb{R}} .
$$

In general, $\underline{H}$ and $\rho$ cannot be selected independently [9].

Let $\underline{\underline{H}}=W \operatorname{diag}(\underline{H}) W^{-1}$ be the so-named Hurst matrix. Then, the stationary-increment stochastic process $Y$ satisfies the bivariate self-similarity relation, $\forall a>0$ :

$$
\begin{aligned}
\left\{Y_{1}^{\underline{H}}, \Sigma_{X}, P W\right. & \left.(t), Y_{2}^{\underline{H}, \Sigma_{X}, W}(t)\right\}_{t \in \mathbb{R}} \stackrel{\text { fdd }}{\asymp} \\
& \left\{a \underline{\underline{H}}\left(Y_{1}^{\underline{H}}, \Sigma_{X}, W(t / a), Y_{2}^{\underline{H}}, \Sigma_{X}, W(t / a)\right)\right\}_{t \in \mathbb{R}},
\end{aligned}
$$

where $\stackrel{\text { fdd }}{\simeq}$ stands for the convergence of finite dimensional distributions and $a \underline{\underline{H}} \triangleq \sum_{k=0}^{+\infty} \log ^{k}(a) \underline{H}^{k} / k$ !.

Wavelet estimation of $\underline{\mathbf{H}}$. When the mixing matrix $W$ is diagonal, the estimation of $\underline{H}$ can be conducted by means of univariatelike approaches $[17,22]$. Otherwise, i.e., when $W$ is non-diagonal, such approaches yield dramatically poor estimates. In $[4,11]$, an intrinsically multivariate wavelet-based procedure is proposed and studied. It relies on the multivariate discrete wavelet transform coefficients $\left(D\left(2^{j}, k\right)\right) \equiv D_{Y}\left(2^{j}, k\right)=\left(D_{Y_{1}}\left(2^{j}, k\right), D_{Y_{2}}\left(2^{j}, k\right)\right)$, $\forall k \in \mathbb{Z}, \forall j \in\left\{j_{1}, \ldots, j_{2}\right\}$, with $\forall m \in\{1,2\}: D_{Y_{m}}\left(2^{j}, k\right)=$ $\left\langle 2^{-j / 2} \psi_{0}\left(2^{-j} t-k\right) \mid Y_{m}(t)\right\rangle[23,24]$.

The sample wavelet spectrum, or variance, at octave $j$, is defined as the $2 \times 2$ symmetric positive semidefinite random matrix

$$
S\left(2^{j}\right)=\frac{1}{n_{j}} \sum_{k=1}^{n_{j}} D\left(2^{j}, k\right) D\left(2^{j}, k\right)^{*}, \quad n_{j}=\frac{N}{2^{j}},
$$

where $N$ is the sample size. Let $\left\{\lambda_{1}\left(2^{j}\right), \lambda_{2}\left(2^{j}\right)\right\}$ be the eigenvalues of $S\left(2^{j}\right)$. The estimator $\left(\hat{H}_{1}, \hat{H}_{2}\right)$ of $\left(H_{1}, H_{2}\right)$ is defined by means of weighted $\log$-regressions across scales $2^{j_{1}}, \ldots, 2^{j_{2}}[4,11]$ :

$$
\hat{H}_{m}=\left(\sum_{j=j_{1}}^{j_{2}} w_{j} \log _{2} \lambda_{m}\left(2^{j}\right)\right) / 2-\frac{1}{2}, \quad \forall m=1,2,
$$

where $w_{j}$ are linear regression weights. Under mild assumptions [4, 11], it can be shown that $\lambda_{m}\left(2^{j}\right) \stackrel{P}{\sim} C_{m} 2^{j\left(2 H_{m}+1\right)}, \forall m=1,2$, for some $C_{m}>0$ as $j, N \rightarrow \infty$, and thus that $\left(\hat{H}_{1}, \hat{H}_{2}\right) \stackrel{P}{\rightarrow}\left(H_{1}, H_{2}\right)$.

\section{TIME IRREVERSIBILITY AND DELAYS IN BIVARIATE SCALE FREE DYNAMICS: MODELING AND ANALYSIS}

\subsection{Model}

Technically, the broad definition of ofBm proposed in [9] permits defining exactly self-similar, time irreversible processes. Nevertheless, modeling time irreversibility caused by pure delays amongst
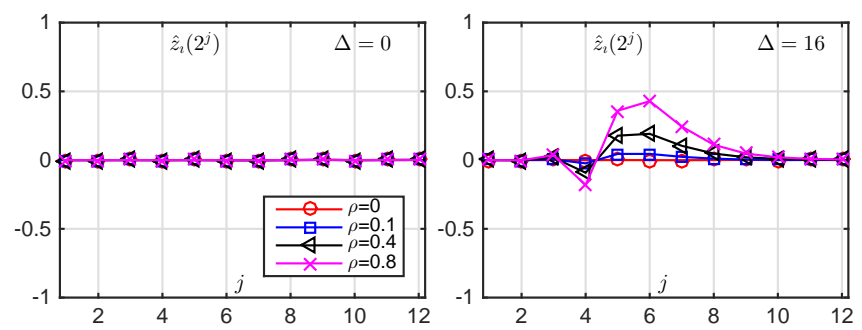

Fig. 1. $z$-score of imaginary wavelet coherence.Monte Calo averages for several $\rho$ and for $\Delta=0$ (left) and $\Delta=16$ (right).

components requires departing from exact self-similarity. Yet, this preserves the intuition of scale-free dynamics via the notion of asymptotic self-similarity and addresses the practical needs involved in the modeling of real-world data, particularly from neuroscience. A time irreversible model $Y$ can be defined as an ofBm as in definition (1) where the stationary increments of component $X_{2}$ are delayed in time by $\Delta>0$. It is easy to show that $Y$ is asymptotically self-similar in the sense that the key relation (2) holds in the coarse scale limit $0<a \rightarrow \infty$. Yet, a delay $\Delta>0$ introduces bias in the wavelet eigenvalue-based estimation of $\underline{H}$ as defined by (4) when $j_{1}<\log _{2}(\Delta)$. Hence, it is necessary to both reliably detect the presence delays and jointly estimate delays and $\underline{H}$.

\subsection{Detection of delays and estimation of $\Delta$ and $\underline{H}$}

Complex Wavelets. Delayed information is classically obtained by inspection of the phase of a complex bivariate representation. Therefore, we make use of a complex-valued discrete wavelet transform (instead of the real-valued one described above). Hereinafter, we use a $\mathbb{C}$-valued mother wavelet function $\psi_{0}$, with $N_{\psi} \in \mathbb{N}$ vanishing moments, produced by the $q$-shift construction proposed in $[25,26]$. From the now complex-valued wavelet coefficients $\left(D\left(2^{j}, k\right)\right) \equiv D_{Y}\left(2^{j}, k\right)=\left(D_{Y_{1}}\left(2^{j}, k\right), D_{Y_{2}}\left(2^{j}, k\right)\right)$, we compute the wavelet spectrum $\left\{S\left(2^{j}\right)\right\}$ as defined by (3) above, whose non-diagonal entries are potentially complex-valued. The complex wavelet coherence function is defined by

$$
\hat{\gamma}\left(2^{j}\right)=S_{12}\left(2^{j}\right) /\left(S_{11}\left(2^{j}\right) S_{22}\left(2^{j}\right)\right)^{\frac{1}{2}},
$$

where $\hat{\gamma}_{\imath}\left(2^{j}\right)=\Im\left(\hat{\gamma}\left(2^{j}\right)\right)$ denotes its imaginary part [27].

Fisher transform. The Fisher $z$-transform of $\hat{\gamma}_{\imath}\left(2^{j}\right)$ is given by

$$
\hat{z}_{2}\left(2^{j}\right)=\frac{1}{2} \log \left(\frac{1+\hat{\gamma}_{\imath}\left(2^{j}\right)}{1-\hat{\gamma}_{\imath}\left(2^{j}\right)}\right)
$$

and analogously so for its population counterpart $z_{\imath}\left(2^{j}\right)$. Under mild conditions on $Y$ and as a consequence of the decorrelation property of the wavelet transform [28], $\hat{z}_{\imath}\left(2^{j}\right)$ is approximately Gaussian with mean zero when $\gamma_{2}\left(2^{j}\right)=0$. For the Fisher $z$-transform of the modulus of $\hat{\gamma}\left(2^{j}\right)$, the variance is known to approach asymptotically $\frac{1}{n_{j}-3}$. However, this is not the case when considering only the imaginary part (cf. Section 4 below). For this reason, we propose estimating the standard deviation of $\hat{z}_{\imath}\left(2^{j}\right)$ by means of a bootstrap procedure.

Multivariate wavelet domain bootstrap. To approximate the distribution of $\hat{z}_{\imath}\left(2^{j}\right)$, a wavelet-domain bootstrap method can be constructed that preserves the joint covariance structure of the wavelet coefficients. To this end, rather than bootstrapping independently on the wavelet coefficients of each component, the 

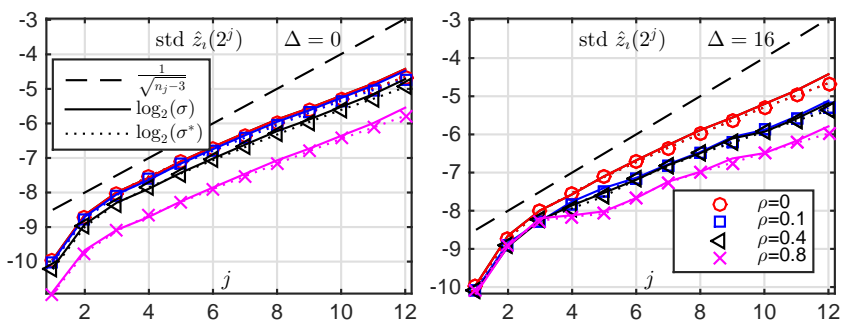

Fig. 2. Standard deviation of $\hat{z}_{\imath}\left(2^{j}\right)$. Bootstrap estimates compared to Monte Carlo ensemble average and to the classical approximation $\frac{1}{\sqrt{n_{j}-3}}$, for several $\rho$ and for $\Delta=0$ (left) and 16 (right).

vector coefficients $D\left(2^{j}, k\right), k=1, \ldots, n_{j}$, are used in a (circular) block-bootstrap procedure [29]. For each scale $j$, from the periodically extended samples $\left(D\left(2^{j}, 1\right), \ldots, D\left(2^{j}, n_{j}\right)\right), R$ block bootstrap resamples $D_{j}^{*(r)}=\left(D^{*(r)}\left(2^{j}, 1\right), \ldots, D^{*(r)}\left(2^{j}, n_{j}\right)\right)$, $r=1, \ldots, R$, are generated by a drawing-with-replacement procedure of $\left\lceil\operatorname{card}(Y) / L_{B}\right\rceil$ overlapping blocks of fixed size $L_{B}$, $\left(D\left(2^{j}, k\right), \ldots, D\left(2^{j}, k+L_{B}-1\right), k=1, \ldots, n_{j}\right.$. Then, for each resample $D_{j}^{*(r)}$, bootstrap estimates $S^{*(r)}(j)$ and $\hat{z}_{\imath}^{*(r)}\left(2^{j}\right)$ are computed, whence the bootstrapped standard deviation $\sigma_{z_{2}}^{*}$ of the latter is obtained.

Delay detection. Let $\Phi$ be the standard normal cumulative distribution function and define $t_{\pi}=\Phi^{-1}(\pi)$. The bootstrap test for $\hat{z}_{\imath}\left(2^{j}\right)=0$ for a single scale $2^{j}$ is defined by

$$
d_{\alpha}\left(2^{j}\right)=\left\{\begin{array}{lll}
1: & p\left(2^{j}\right)<\alpha & \left(z_{\imath}\left(2^{j}\right)=0 \text { rejected }\right) \\
0: & p\left(2^{j}\right) \geq \alpha & \left(z_{\imath}\left(2^{j}\right)=0 \text { accepted }\right)
\end{array}\right.
$$

where $p\left(2^{j}\right)=2 \min \left(\Phi\left(\hat{z}_{\imath}\left(2^{j}\right) / \sigma_{z_{\imath}}^{*}\right), 1-\Phi\left(\hat{z}_{\imath}\left(2^{j}\right) / \sigma_{z_{\imath}}^{*}\right)\right)$ is the pvalue of $\hat{z}_{\imath}\left(2^{j}\right)$. Furthermore, over multiple scales $j=j_{1}, \ldots, j_{2}$, the bootstrap tests are corrected for multiple hypotheses using a false discovery rate (FDR) adjustment, which effectively defines an adjusted significance $\alpha_{\text {FDR }}$. The test for non-zero delay $\Delta$ is then formulated based on the adjusted test decisions for the multiple scales $j=j_{1}, \ldots, j_{2}$ as

$$
d_{\alpha}^{\Delta}=\left\{\begin{array}{l}
1: \exists j \in\left(j_{1}, j_{2}\right): d_{\alpha_{\mathrm{FDR}}}\left(2^{j}\right)=1(\Delta=0 \text { rejected }) \\
0: \forall j \in\left(j_{1}, j_{2}\right): d_{\alpha_{\mathrm{FDR}}}\left(2^{j}\right)=0(\Delta=0 \text { accepted }) .
\end{array}\right.
$$

Delay estimation. When a non-zero delay is detected, an estimate of the delay $\log _{2}(\hat{\Delta})$ is obtained as the median minus 1 of the set of the up to 5 finest scales for which $p\left(2^{j}\right)<\hat{\alpha}_{\mathrm{FDR}}, j=j_{1}, \ldots, j_{2}$. Here, the choice of the median is natural for making the estimator robust. Subtracting one compensates for the finite frequency selectivity of the wavelet, and the use of at most five detected non-zero scales counteracts the increased number of detected scales when $\rho$ is increasing, cf., Fig. 3, bottom row. Numerical experiments reported in Sec. 4 assess the robustness of the estimator with respect to $\rho$ and $\Delta$.

Estimation of $\underline{H}$. To arrive at a consistent estimator for $\underline{H}$ for $\Delta>$ 0 , the linear regression (4) must be modified. First, the octaves

$$
\check{j}=\left(\log _{2}(\hat{\Delta}), \log _{2}(\hat{\Delta})+1\right)
$$

are excluded. Second, the presence of delay induces a vertical offset of $\lambda_{1}\left(2^{j}\right)$ for scales $2^{j}<\Delta$, cf. Fig. 5. This makes it necessary to use two independent intercept parameters (for $j<\log _{2}(\Delta)$ and
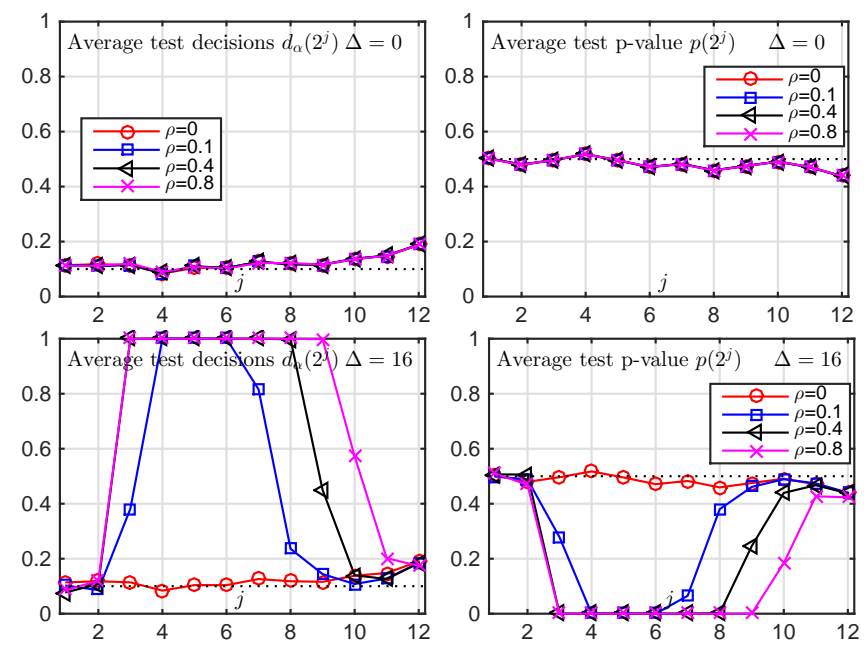

Fig. 3. Bootstrapped based delay detection. Bootstrapped test decisions (left) and p-values (right) averaged over 500 realizations, for $\Delta=0$ (top) and $\Delta=16$ (bottom, test significance $\alpha=0.1$ ).

$j>\log _{2}(\Delta)$ ). Tedious but straightforward calculations, not reported here, lead to modified linear regression weights $\tilde{w}_{j}$,

$$
\hat{H}_{m}^{(b i)}=\left(\sum_{j \in\left(j_{1}, j_{2}\right) \backslash \grave{j}} \tilde{w}_{j} \log _{2} \lambda_{m}\left(2^{j}\right)\right) / 2-\frac{1}{2}, \quad \forall m=1,2 .
$$

By similar arguments to those in $[4,11]$, it can be shown under mild assumptions that the estimator (7) is consistent.

\section{STATISTICAL PERFORMANCE ASSESSMENT}

Simulation setup. To assess the detection and estimation performance of the procedures proposed above, Monte Carlo simulations were conducted using $N_{\mathrm{MC}}=500$ independent realizations of the model $Y$ as described in Sec. 3.1. The path length was $N=2^{18}$ with parameter values $H=(0.4,0.6), \Sigma_{X}=\left(\begin{array}{ll}1 & \rho \\ \rho & 1\end{array}\right)$ for multiple values of $\rho$, and $W$ orthogonal matrices drawn independently for each realization. A $q$-shift complex wavelet of length $N_{F}=10$ was used. A total of $R=500$ bootstrap replicates were generated, with a block size $L_{B}=8$. Bootstrap test significance and FDR level, respectively, were set to $\alpha=0.1$.

Fisher $z$-transform of the imaginary wavelet coherence $\hat{z}_{\imath}\left(2^{j}\right)$. Fig. 1 (left) displays averages of $\hat{z}_{\imath}\left(2^{j}\right)$ for $\rho=(0,0.2,0.8)$ and illustrates the fact that the imaginary part of the wavelet coherence is identically zero for all scales when $\Delta=0$ or when $\rho=0$. To the converse, it also takes on non-zero values when $\Delta \neq 0$ and $\rho \neq 0$ at octaves $j$ in a neighborhood of $\log _{2}(\Delta)$. This confirms that the imaginary part of the wavelet coherence can be used in the detection and estimation of delays.

Scale-wise bootstrap test for non-zero $\hat{z}_{\imath}\left(2^{j}\right)$. Fig. 2 reports estimates of the standard deviation $\sigma_{z_{2}}^{*}$. Averages over bootstrapped standard deviation estimates (dotted line with symbols) are compared to averages over Monte Carlo estimates, acting as ensemble averages (solid lines), as well as the classical approximation $\frac{1}{\sqrt{n_{j}-3}}$ (dashed line). The bootstrap variance estimates are observed to be accurate whatever the correlation level $\rho$, and to be much closer to 

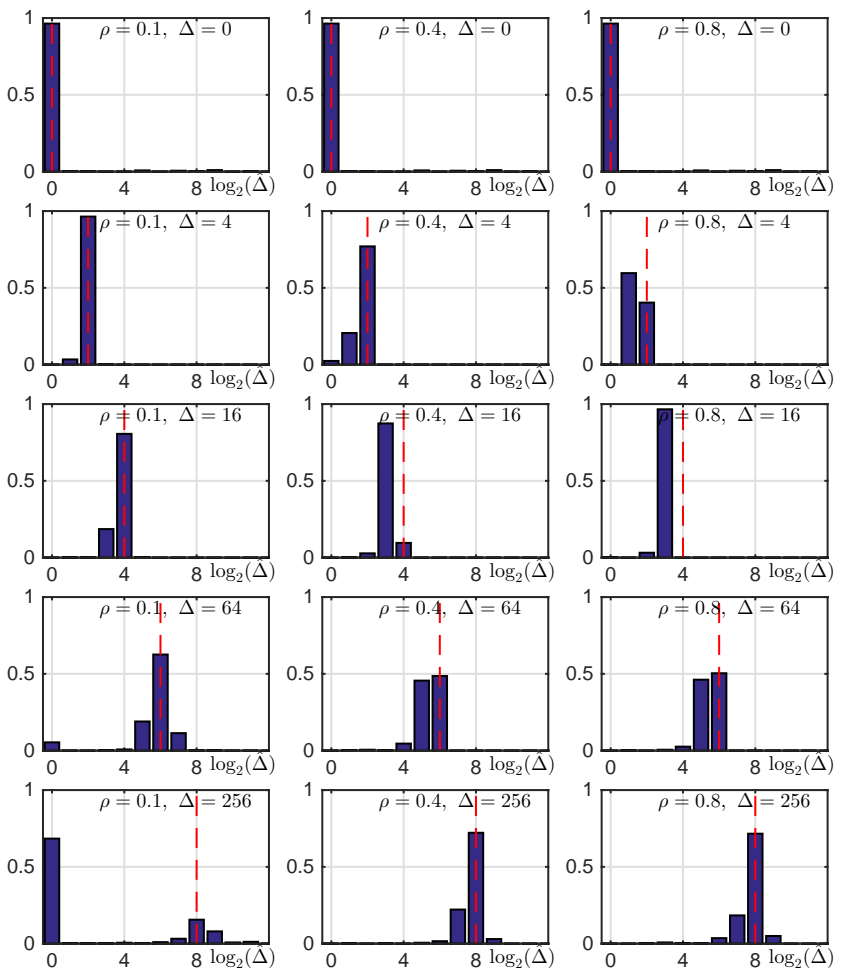

Fig. 4. Delay estimation performance as functions of $\log _{2} \hat{\Delta}$ for different values of $\rho$ (left to right) and $\Delta$ (top to bottom). True $\Delta$ : dashed red vertical line.

the Monte Carlo ensemble values than the approximation $\frac{1}{\sqrt{n_{j}-3}}$. Variances are slightly underestimated at largest scales only, when $n_{j}$ and $L_{B}$ are of the same order of magnitude.

Fig. 3 displays average test decision (left column) and p-values (right column) of the bootstrap method for the detection of non-zero coherence as a function of scale $j$ for $\Delta=0$ (top row) and $\Delta=16$ (bottom row) and $\rho=(0,0.2,0.8)$. The results show that when $\Delta=0$ or $\rho=0$ (i.e., under the null hypothesis), average test decisions are close to the preset nominal value $\alpha=0.1$, and that the average $p$-value is close to 0.5 . Thus, the proposed test procedure satisfactorily reproduces the null distribution. When $\Delta \neq 0$ and $\rho \neq 0$ (i.e., under the alternative hypothesis), the test detects the non-zero values of the coherence for a range of scales $j$ including $\log _{2}(\Delta)$. The size of this neighborhood is observed to decrease with decreasing $\rho$. This is due to the decreasing average value and increasing variance for the imaginary coherence, cf. Fig. 1. Yet, satisfactorily, even at law correlation $\rho=0.2$, the test always rejects the null hypothesis for a range of scales $j$.

Delay estimation. Fig. 4 reports histograms of $\log _{2}(\hat{\Delta})$. It shows that, when the null hypothesis $\Delta=0$ holds, the proposed procedure rejects it in about $4 \%$ of the cases, in close agreement with the preset choices $\alpha=0.1, q=0.1$. On the other hand, when $\Delta \neq 0$, the proposed procedure rejects $\Delta=0$ in nearly all cases, thus showing a very high power. The only exception is when $\rho$ (and, therefore, the imaginary part of the coherence) is small and $\Delta$ is large (whence the imaginary coherence is non-zero only at large scales $j$, in turn associated with large variances; cf. Fig. 1). This leads to lower power (see Fig. 4, bottom left). Once a non-zero $\Delta$ is detected, though, its estimates are accurate.
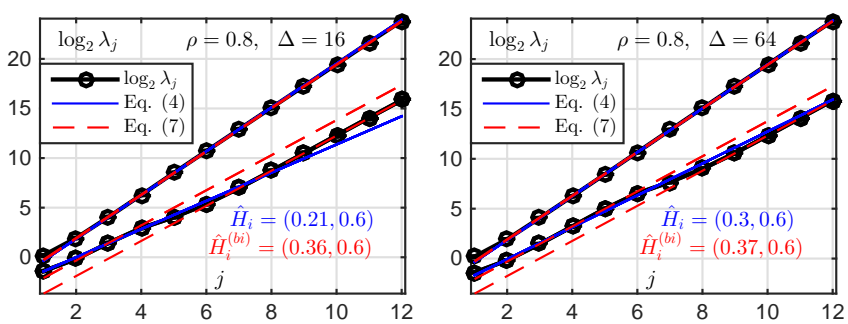

Fig. 5. Functions $\log _{2} \lambda_{j}$ (Monte Carlo averages) for $\Delta=16$ (left) and $\Delta=64$ (right), with average estimates $\hat{H}_{m}$ from standard linear regression as in (4), versus newlt defined estimates $\hat{H}_{m}^{(b i)}$ as in (7).

\begin{tabular}{|l||r|r|r|r|r|r|}
\hline \multicolumn{1}{|l||}{ unweighted } & \multicolumn{3}{c|}{ rmse for $\hat{H}_{1}$} & \multicolumn{3}{c|}{ rmse for $\hat{H}_{1}^{(b i)}$} \\
\hline$\rho$ & 0.10 & 0.40 & 0.80 & 0.10 & 0.40 & 0.80 \\
\hline$\Delta=4$ & $\mathbf{0 . 0 1 0}$ & $\mathbf{0 . 0 1 1}$ & 0.031 & 0.012 & 0.013 & $\mathbf{0 . 0 1 4}$ \\
$\Delta=16$ & $\mathbf{0 . 0 1 0}$ & 0.020 & 0.095 & 0.017 & $\mathbf{0 . 0 1 7}$ & $\mathbf{0 . 0 2 7}$ \\
$\Delta=64$ & $\mathbf{0 . 0 1 0}$ & 0.026 & 0.126 & 0.023 & $\mathbf{0 . 0 2 5}$ & $\mathbf{0 . 0 4 3}$ \\
$\Delta=256$ & $\mathbf{0 . 0 1 0}$ & 0.024 & 0.113 & 0.012 & $\mathbf{0 . 0 1 6}$ & $\mathbf{0 . 0 3 1}$ \\
\hline \hline weighted & \multicolumn{3}{|c|}{ rmse for $\hat{H}_{1}$} & \multicolumn{3}{c|}{ rmse for $\hat{H}_{1}^{(b i)}$} \\
\hline$\rho$ & 0.10 & 0.40 & 0.80 & 0.10 & 0.40 & 0.80 \\
\hline$\Delta=4$ & 0.010 & 0.021 & 0.110 & $\mathbf{0 . 0 0 6}$ & $\mathbf{0 . 0 0 7}$ & $\mathbf{0 . 0 2 8}$ \\
$\Delta=16$ & 0.011 & 0.041 & 0.193 & $\mathbf{0 . 0 1 0}$ & $\mathbf{0 . 0 1 5}$ & $\mathbf{0 . 0 4 5}$ \\
$\Delta=64$ & $\mathbf{0 . 0 1 0}$ & 0.026 & 0.100 & 0.017 & $\mathbf{0 . 0 1 9}$ & $\mathbf{0 . 0 3 7}$ \\
$\Delta=256$ & $\mathbf{0 . 0 0 9}$ & 0.015 & 0.040 & 0.010 & $\mathbf{0 . 0 1 2}$ & $\mathbf{0 . 0 1 5}$ \\
\hline
\end{tabular}

Table 1. Estimation performance for the smaller exponent $H_{1}$. Rmse values as a function of $\rho$ and $\Delta$ : standard estimator (4) (left) and proposed estimator (7) (right, best results marked in bold).

Estimation of $H$. Fig. 5 plots Monte Carlo averages of $\lambda_{j}$. It illustrates the change of intercept between fine and coarse scales for the component with smaller exponent $H_{1}$, which induces the aforementioned bias in $\hat{H}_{1}$ for the standard estimator (4). Table 1 compares the root mean squared error (rmse) of $\hat{H}_{1}$ and of the proposed consistent estimator $\hat{H}_{1}^{(b i)}$ defined by (7). It clearly shows that the use of (7), which takes into account the estimated delay $\Delta$, is beneficial, and significantly so when $\rho$ is large (rmse values divided by up to 4$)$. When $\rho$ is small, (7) leads to slightly larger rmse values than (4), since the increase in variance caused by excluding scales for (7) outweighs the larger bias of (4).

\section{CONCLUSIONS}

In this paper, the first framework for the modeling, detection and estimation of delay parameters as well as for the estimation of selfsimilarity parameters is constructed. It assumes a single multivariate time series is available, and is based on an original combination of $\mathbb{C}$-valued wavelets, bootstrapping and wavelet eigenanalysis. Monte Carlo experiments show that the proposed protocols display high delay detection probability as well as accurate delay and scale-free parameters estimation for realistic sample sizes and correlation levels.

Future work includes the extension of the proposed methods to the detection and estimation of delays not restricted to dyadic values and the construction of procedures for the detection and estimation of delay when multiple components are present. Applications in the analysis of neuroscientific multivariate time series data are also under investigation. 


\section{REFERENCES}

[1] G. Samorodnitsky and M. Taqqu, Stable non-Gaussian random processes, Chapman and Hall, New York, 1994.

[2] D. Veitch and P. Abry, "A wavelet-based joint estimator of the parameters of long-range dependence," IEEE Trans. Info. Theory, vol. 45, no. 3, pp. 878-897, 1999.

[3] H. Wendt, P. Abry, and S. Jaffard, "Bootstrap for empirical multifractal analysis," IEEE Signal Processing Mag., vol. 24, no. 4, pp. 38-48, 2007.

[4] P. Abry and G. Didier, "Wavelet eigenvalue regression for $n$ variate operator fractional Brownian motion," Journal of Multivariate Analysis, vol. 168, pp. 75-104, November 2018.

[5] B. B. Mandelbrot and J. W. van Ness, "Fractional Brownian motion, fractional noises and applications," SIAM Reviews, vol. 10, pp. 422-437, 1968.

[6] P. Flandrin, "Wavelet analysis and synthesis of fractional Brownian motion," IEEE Trans. on Info. Theory, vol. IT-38, no. 2, pp. 910-917, 1992.

[7] M. Maejima and J. D. Mason, "Operator-self-similar stable processes," Stochastic Processes and their Applications, vol. 54, no. 1, pp. 139-163, 1994.

[8] J. D. Mason and Y. Xiao, "Sample path properties of operatorself-similar Gaussian random fields," Theory of Probability \& Its Applications, vol. 46, no. 1, pp. 58-78, 2002.

[9] G. Didier and V. Pipiras, "Integral representations and properties of operator fractional Brownian motions," Bernoulli, vol. 17, no. 1, pp. 1-33, 2011.

[10] G. Didier and V. Pipiras, "Exponents, symmetry groups and classification of operator fractional Brownian motions," Journal of Theoretical Probability, vol. 25, pp. 353-395, 2012.

[11] P. Abry and G. Didier, "Wavelet estimation for operator fractional Brownian motion," Bernoulli, vol. 24, no. 2, pp. 895928, 2018.

[12] P. Abry, G. Didier, and H. Li, "Two-step wavelet-based estimation for Gaussian mixed fractional processes," To appear in Statistical Inference for Stochastic Processes, pp. 1-61, 2018.

[13] G. Nolte, O. Bai, L. Wheaton, Z. Mari, S. Vorbach, and M. Hallett, "Identifying true brain interaction from EEG data using the imaginary part of coherency," Clin. Neurophysiol., vol. 115, no. 10, pp. 2292-2307, 2004.

[14] R. Leonarduzzi, P. Abry, S. G. Roux, H. Wendt, S. Jaffard, and S. Seuret, "Multifractal characterization for bivariate data," in Proc. Eur. Signal Proc. Conf. (EUSIPCO), Rome, Italy, Sept. 2018.

[15] P. Robinson, "Multivariate Local Whittle estimation in stationary systems," Annals of Statistics, vol. 36, no. 5, pp. 25082530, 2008.

[16] C. Baek, S. Kechagias, and V. Pipiras, "Asymptotics of bivariate local Whittle estimators with applications to fractal connectivity," Preprint, 2018.

[17] P.-O. Amblard and J.-F. Coeurjolly, "Identification of the multivariate fractional Brownian motion," IEEE Trans. Signal Proces., vol. 59, no. 11, pp. 5152-5168, 2011.

[18] J.-F. Coeurjolly, P.-O. Amblard, and S. Achard, "Wavelet analysis of the multivariate fractional Brownian motion," ESAIM: Probability and Statistics, vol. 17, pp. 592-604, 2013.
[19] S. Achard, D. S. Bassett, A. Meyer-Lindenberg, and E. Bullmore, "Fractal connectivity of long-memory networks," Phys. Rev. E, vol. 77, no. 3, pp. 036104, 2008.

[20] S. Achard and I. Gannaz, "Multivariate wavelet Whittle estimation in long-range dependence," Journal of Time Series Analysis, vol. 37, no. 4, pp. 476-512, 2016.

[21] I. Gannaz, S. Achard, M. Clausel, and F. Roueff, "Analytic wavelets for multivariate time series analysis," in Wavelets and Sparsity XVII, 2017, vol. 10394, p. 103941X.

[22] H. Wendt, G. Didier, S. Combrexelle, and P. Abry, "Multivariate Hadamard self-similarity: testing fractal connectivity," Physica D, vol. 356-357, pp. 1-36, 2017.

[23] I. Daubechies, Ten Lectures on Wavelets, vol. 61, Society for Industrial and Applied Mathematics, Philadelphia-PA, 1992.

[24] S. Mallat, A Wavelet Tour of Signal Processing, Academic Press, San Diego, CA, 1998.

[25] N.G. Kingsbury, "A dual-tree complex wavelet transform with improved orthogonality and symmetry properties," in Proc. IEEE Int. Conf. Image Processing, vol. 2, 2000, pp. 375-378.

[26] I. Selesnick, R. Baraniuk, and N. Kingsbury, "The dual-tree complex wavelet transform," IEEE Signal Processing Magazine, vol. 22, no. 6, pp. 123-151, 2005.

[27] B. Whitcher, P. Guttorp, and D. B. Percival, "Wavelet analysis of covariance with application to atmospheric time series," J. Geophys. Research: Atmospheres, vol. 105, no. D11, pp. 14941-14962, 2000.

[28] P. Abry, P. Flandrin, M. S. Taqqu, and D. Veitch, "Selfsimilarity and long-range dependence through the wavelet lens," in Theory and applications of long-range dependence, pp. 527-556. Birkhäuser, 2003.

[29] S. N. Lahiri, Resampling Methods for Dependent Data, Springer, New York, 2003. 